\title{
A Prototype Detector for Directional Measurement of the Cosmogenic Neutron Flux
}

\author{
J. Lopez, K. Terao, J.M. Conrad, D. Dujmic, L. Winslow \\ Physics Dept., Massachusetts Institute of Technology, Cambridge, MA 02139
}

\begin{abstract}
This paper describes a novel directional neutron detector. The low pressure time projection chamber uses a mix of helium and $\mathrm{CF}_{4}$ gases. The detector reconstructs the energy and angular distribution of fast neutron recoils. This paper reports results of energy calibration using an $\alpha$ source and angular reconstruction studies using a collimated neutron source. The best performance is obtained with a $12.5 \% \mathrm{CF}_{4}-87.5 \% \mathrm{He}$ gas mixture. At low energies the target for fast neutrons transitions is primarily helium, while at higher energies, the fluorine contributes as a target. The reconstruction efficiency is both energy and target dependent. For neutrons with energies less than $20 \mathrm{MeV}$, the reconstruction efficiency is $\sim 40 \%$ for fluorine recoils and $\sim 60 \%$ for helium recoils.
\end{abstract}

\section{Introduction}

Precise measurement of the energy and direction of cosmogenic-muon-induced fast neutrons, with various levels of underground shielding, will be valuable for future neutrino and dark matter experiments [1. In this paper, we present a first-generation detector for this purpose. The detector design is based on that for the directional dark matter search DMTPC [2. We introduce modest modifications for directional neutron detection 3 . Our first application will be in the Double Chooz (DC) neutrino experiment 4, and it is, therefore, called DCTPC.

DCTPC is a low pressure time projection chamber (TPC) filled with two gasses: (1) He, the fast neutron target, and (2) $\mathrm{CF}_{4}$, a scintillant and a quencher for the electron avalanche. This detector is blind to $\mathrm{x}$-rays and minimum ionizing particles (MIP), like muons, because the density of primary ionization is too small to be detected. The ionization electrons from a non-MIP particle track drift down to a stainless steel ground mesh. Between the ground mesh and the anode, an avalanche of electrons occurs. A charge-coupled device (CCD) camera installed in the detector images the visible scintillation light from the avalanche. The charge created in the avalanche is readout from the anode plate and ground mesh. The track direction and energy can be reconstructed using the CCD image and the charge information. The reconstructed track from the 
recoiling nucleus can then be correlated to the incoming neutron direction and energy. In this article we present on-surface measurements from a small 2.8 liter, first-generation detector for an $\alpha$-source run and a ${ }^{252} \mathrm{Cf}$ neutron source run as well as an on-surface cosmogenic run.

\section{Motivation for DCTPC}

DCTPC provides a benchmark for the Double Chooz fast neutron simulation at the specific sites of the two Double Chooz neutrino detectors 44. The detectors sites have 114 and 300 meters water equivalent (m.w.e) shielding, respectively. The final DCTPC design calls for a large detector located at each site. The small first-generation detector described here will be installed in the 300 m.w.e site for initial studies. The neutron flux energy and angle measurements from the rock can be used to tune the Monte Carlo and demonstrate a clear understanding of this background in the Double Chooz neutrino oscillation analysis. However, the results will provide wider benefits as backgrounds from fast neutrons produced in rock are an issue for all low background experiments.

Low energy neutrons arise from $(\alpha, \mathrm{n})$ interactions due to natural radioactivity in the shielding rock. At locations with low shielding, this neutron source is overwhelmed by neutron production from cosmic ray muons. However, muons are attenuated with depth and the $(\alpha, \mathrm{n})$ background becomes significant at 300 m.w.e. 5]. DCTPC, with its very low energy threshold, can sample these $(\alpha, \mathrm{n})$ events. The comparison of the 114 m.w.e. to 300 m.w.e. depths at Double Chooz will be useful in isolating the $(\alpha, \mathrm{n})$ from the muon-induced neutrons.

The remaining two broad categories of neutron production are caused by cosmic ray muon interactions with rock: stopped muon capture and deep inelastic interactions. The rates depend upon the depth of the final experiment because low energy muons are attenuated with shielding. DCTPC sensitivity will reach to $>20 \mathrm{MeV}$ in fast neutron reconstruction, overlapping with the KARMEN2 (surface) and LVD (3200 m.w.e.) data sets. As we show below, the maximum reconstructed recoil energy depends upon the size of the detector.

The FLUKA [6, 7] and GEANT4 [8, 9] simulations are generally used to predict $>10 \mathrm{MeV}$ fast neutrons that are produced by muons. DCTPC adds data points at two depths which will complement the existing measurements. Information on the energy spectra of fast neutrons is scarce, coming only from KARMEN2 experiment on the surface [10, KamLAND at 2700 m.w.e. [1] and the LVD experiment at 3200 m.w.e. 12. DCTPC data will fill the gap in depth between KARMEN2 and deeper experiments. DCTPC also provides an angular distribution of events. In this paper, we show the quality of this angular reconstruction. The unique directional information can help disentangle different neutron sources.

\section{The First Generation Detector}

We have developed a first generation of DCTPC. The TPC has a drift volume above an amplification plane that has two active regions. The amplification 


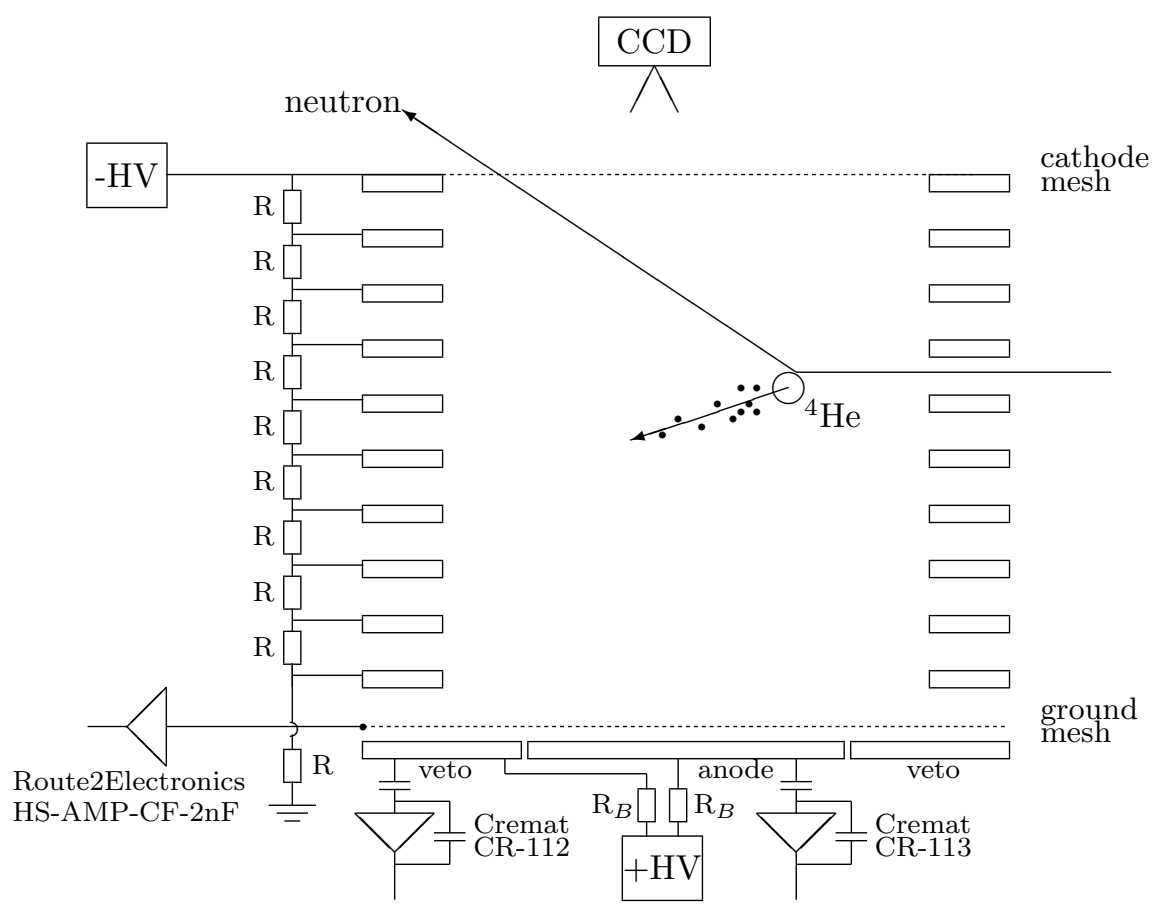

Figure 1: A schematic of the detector: the drift field is created by a cathode mesh, field-shaping rings attached to a resistor chain, and a ground mesh. Primary ionization from a recoiling nucleus drifts down to the ground mesh. The high-field amplification region is formed by the ground mesh and the anode plane. The ground mesh is read out with a fast amplifier and the veto and anode are readout with charge-sensitive preamplifiers. Scintillation light from the amplification region is recorded with the CCD camera.

plane is imaged with one CCD, and the charge is read out for each active region. The redundancy in the readout of the amplification plane is powerful for event reconstruction and background rejection. The detector schematic is shown in the Fig. 1 and a photograph of the detector vessel is shown in Fig. 2.

\subsection{The Time Projection Chamber}

The detector follows the classic design of TPCs. We have chosen a mixture of $\mathrm{CF}_{4}$ and $\mathrm{He}$ as our operating gas. We study several gas ratios below, but most data were taken with the gasses mixed at 75 Torr and 525 Torr respectively. Helium and fluorine both have large cross sections for fast neutron scattering 13. The ${ }^{4} \mathrm{He}$ nucleus is closer to the neutron mass, and therefore the recoils will produce longer tracks than the fluorine nucleus.

Within the vessel, a uniform drift field is molded by a series of copper rings with an inner diameter of $26.9 \mathrm{~cm}$. The rings are connected with resistors, see Fig. 1. The top ring carries a high transparency stainless-steel mesh with wire pitch of $512 \mu \mathrm{m}$ and wire diameter of $31 \mu \mathrm{m}$. This top mesh is maintained at a negative high voltage. The total height of the field cage is $10 \mathrm{~cm}$. Primary ionization electrons are drifted down to a grounded stainless-steel mesh with wire pitch of $256 \mu \mathrm{m}$ and wire diameter of $30 \mu \mathrm{m}$. 

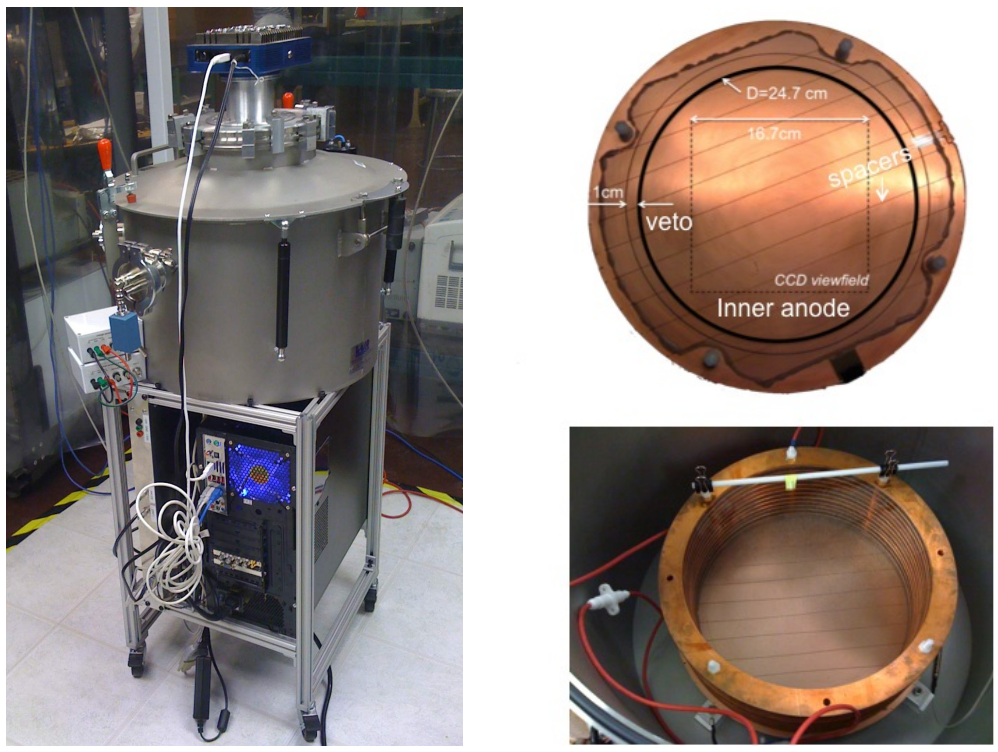

Figure 2: Left: The first generation detector. The detector vessel is sitting on top of the cart which holds the readout electronics. The CCD camera is mounted on the top flange. Right-top: A diagram of the anode plane. Right-bottom: A photograph of the field cage and amplification plane of the TPC. The ground mesh is difficult to see, however the spacers separating it from the anode plane are visible as horizontal lines.

The amplification region is created by the ground mesh and the copper anode that are separated with insulating tubes, called spacers, $440 \mu \mathrm{m}$ in diameter, placed $2.56 \mathrm{~cm}$ apart [14. This high-field amplification gap multiplies the primary ionization charge by a factor of approximately $10^{5}$, as we discuss below.

The anode bias scheme is shown in Fig. 1, where resistor $R_{B}$ is $210 \mathrm{M} \Omega$. The anode of the amplification plane is divided into two regions. The inner part of the anode, diameter $24.7 \mathrm{~cm}$, is used for the detection of tracks. A veto ring, with inner diameter $24.8 \mathrm{~cm}$ and outer diameter $26.8 \mathrm{~cm}$, surrounds the central plane and is used to veto charged particles originating from decays from within the detector components. A central square region, $16.7 \mathrm{~cm} \times 16.7 \mathrm{~cm}$, of the amplification plane is read out optically using the CCD camera. Using this geometry, we calculate that the active volume of the detector that has a charge readout is $5.6 \mathrm{~L}$, while the volume with both CCD and charge readout is $2.8 \mathrm{~L}$.

\subsection{The Gas Mixture and Associated Operating Voltages}

In order to select the gas mixture, we studied three gases: $100 \% \mathrm{CF}_{4}, 12.5 \%$ $\mathrm{CF}_{4}-87.5 \% \mathrm{He}$, and $6.25 \% \mathrm{CF}_{4}-93.75 \% \mathrm{He}$. Operating voltages, listed in Table 1. were optimized for each gas. However, we had difficulty finding suitable operating conditions for the $6.25 \%$ mixture. Small variations in voltage caused very large gain differences. We also found that the absolute and relative energy calibrations were inconsistent over several gas fills with this mixture, likely due 


\begin{tabular}{lccccccc}
\hline $\begin{array}{l}\mathrm{CF}_{4} \\
\%\end{array}$ & $\begin{array}{c}\mathrm{P} \\
{[\text { Torr }]}\end{array}$ & $\begin{array}{c}\mathrm{V}_{\text {anode }} \\
{[\mathrm{V}]}\end{array}$ & $\mathrm{V}_{\text {drift }}$ & $\begin{array}{c}\mathrm{G}_{\mathrm{CCD}} \\
{\left[\mathrm{ADU} / \mathrm{keV}_{\alpha}\right]}\end{array}$ & $\begin{array}{c}\mathrm{G}_{\text {anode }} \\
{\left[\mathrm{mV} / \mathrm{keV}_{\alpha}\right]}\end{array}$ & $\begin{array}{c}\text { Gas } \\
\text { gain }\end{array}$ & $\begin{array}{c}\text { Threshold } \\
{[\mathrm{mV}]}\end{array}$ \\
\hline 100 & 75 & 680 & -1200 & 16.3 & 0.346 & $1.1 \times 10^{5}$ & 20.3 \\
12.5 & 600 & 700 & -1600 & 9.18 & 0.187 & $>6.0 \times 10^{4}$ & 18.8 \\
6.25 & 600 & 600 & -1600 & 15.3 & 0.518 & $>1.6 \times 10^{5}$ & 20.3 \\
\hline
\end{tabular}

Table 1: Summary of detector calibrations for running in the various gas mixtures. We report the run conditions for the $6.25 \%$ mixture here, however due to instability, we do not report on this mixture further (see text). The trigger threshold is that used for neutron and background running. Higher thresholds are needed for the energy calibration runs.

to the much steeper increase of gain with respect to anode voltage. Therefore, we rejected this mixture and do not report the results of the $6.25 \% \mathrm{CF}_{4}$ below.

\section{3. $C C D$ Readout}

The CCD camera records scintillation light created in the amplification region, see Fig. 1. The camera is the U6 model made by Apogee Instruments Inc., with the Kodak KAF-1001E CCD chip. The chip is kept at $-20{ }^{\circ} \mathrm{C}$ to minimize the dark current. An image from the amplification plane is focused to the CCD chip using a Nikon lens, of focal length $55 \mathrm{~mm}$ and set to $\mathrm{f} / 1.2$. The CCD chip consists of 1024 by 1024 square pixels where the side length of a pixel is $24 \mu \mathrm{m}$. The active area on the anode plate is a square of $16.7 \mathrm{~cm} \times 16.7 \mathrm{~cm}$, see Fig. 2. In order to maximize the signal-to-noise ratio, we merge the CCD pixels into $4 \times 4$ bins during readout, so that the effective spatial resolution is $652 \mu \mathrm{m}$. The unit for one pixel count returned by the CCD readout is an ADU (Analog-to-Digital Unit).

The camera does not use a shutter and the CCD sensor is always live, even during the CCD readout. This leads to two types of exposures: a nominal exposure lasting 1 second, and a shorter (typically $250 \mathrm{~ms}$ ) 'parasitic' exposure taken during the CCD readout and event processing. Track images recorded during the short parasitic exposure can be shifted in the direction of readout of CCD rows, and do not have corresponding signal in the charge readout channels. Therefore, they are easy to remove during analysis.

\subsection{Charge Readout}

The charge readout is very powerful in suppressing CCD-specific backgrounds (e.g., direct hits in CCD chip and residual bulk images). This is the major improvement over the CCD-only approach described in Ref. [3]. The anode plate contains three regions separated by electrically insulated gaps, as shown in Fig 2 . The outermost circular region is connected to a common ground, onto which the ground mesh is attached. The inner disk region is the actual anode, maintained at $680 \mathrm{~V}$. The circular-ring region between is used as a veto and is kept at the same potential as the anode.

When a particle enters from outside, its path must cross the veto region. Therefore, we can detect this type of event by inspecting the charge signal from 
the veto region. The veto is not applied at the trigger level. The veto signal is stored for use in the analysis.

The anode signal is collected using a charge-sensitive preamplifier (CSP), the Cremat CR-113 with a gain of $1.5 \mathrm{mV} / \mathrm{pC}$. The pulse height of the output of the CSP corresponds to the total charge collected on the anode. Therefore, the pulse height is used to reconstruct the total energy of the event. The veto electrode is read out through the more sensitive CSP, the Cremat CR-112 with a gain of $15 \mathrm{mV} / \mathrm{pC}$. The ground mesh is read out through a fast current amplifier, the Route2Electronics HS-AMP-CF-2nF with 1 ns rise time and gain of 80. The fast amplifier has a built-in protection that guards against discharges from this high-capacitance detector. The CSPs are protected with $300 \Omega$ resistors. All three signals are digitized using the ATS860, a $250 \mathrm{MS} / \mathrm{s}$ 8-bit flash ADC made by AlazarTech. The raw unit for charge readout in the the following discussion is $\mathrm{mV}$. The digitization of all three channels is triggered by a signal on the anode and stored with the current CCD image. The trigger threshold used is gas dependent.

\subsection{Event Readout, Reconstruction and Correlation}

The combination of the four measurements, CCD image, charge deposited on the inner anode and veto anode and the current recorded at the ground mesh, is used for reconstruction. The CCD track reconstruction starts with subtracting the bias image that is obtained at the beginning of every run. At this time, any unusually high-valued pixels are removed. The image is then smoothed. With the smoothed image, a search is conducted for clusters of adjacent pixels with values $3.7 \sigma$ larger than the mean value of the image 2. If enough such pixels are found, the cluster is determined to be a possible track. An additional ring of pixels around the main cluster is added to account for diffusion, and the track is the corresponding pixels from the original un-smoothed image. For each of these tracks, the energy, range, angle, maximum value, and several moments are calculated.

We require that the track be contained within the TPC. The veto removes signals from radiation emitted from the drift rings or the vessel wall. However, this also vetoes long tracks produced within the active volume that enter the veto. Additional inefficiency comes from the requirement that the track be fully contained in the CCD view-field. We show below that these are not a significant constraints, even in the first generation detector.

Tracks imaged by the CCD camera are matched to charge waveforms using the reconstructed energy. The rate of events in the detector, even with the neutron and $\alpha$ sources, is relatively low, and most exposures contain no tracks.

\section{Calibration}

Calibration of the detector was performed using a ${ }^{241} \mathrm{Am} \alpha$ source. A window on the source degrades the energy of the $\alpha$ particles leading to a mean energy of $4.40 \pm 0.04 \mathrm{MeV}$ [15]. Example $\alpha$ events in two different gas mixtures are shown in Fig. 3 . 


\subsection{Spatial Calibration}

The spatial calibration is obtained by measuring the positions of the wire spacers separating the anode plane from the ground mesh, shown in Fig. 2. The spacers create a region of low gain. A ${ }^{137} \mathrm{Cs}$ source is used to generate electronic recoils so that a small but diffuse amount of light is seen in each image. By integrating many images, the spacers become visible as regions with low light levels. The spacers are placed $2.5 \mathrm{~cm}$ apart, and the fit gives a calibration of $163 \mu \mathrm{m}$ per pixel. The total area of the 1024 by 1024 pixel region imaged by the CCD is then $16.7 \mathrm{~cm} \times 16.7 \mathrm{~cm}$, as discussed in Sec. 3.3 . The uncertainty on the spatial resolution is believed to be dominated by systematics from misplacement of spacers, optical distortions, and deviations in the spacer shape from a line.

\subsection{Energy Calibration}
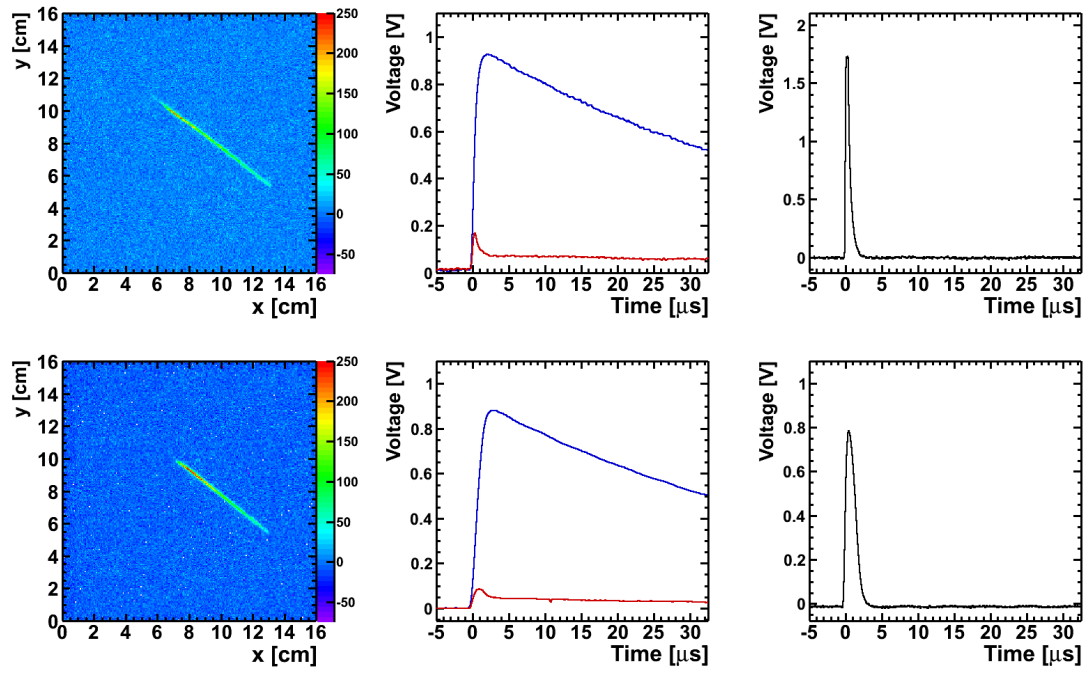

Figure 3: For $\alpha$ particle tracks from the ${ }^{241} \mathrm{Am}$ source, images of the CCD and charge readout system are shown. Left: CCD image of $\alpha$ particle tracks. Middle: Waveforms from the Veto and Anode charge read-out. Right: Waveforms from the Ground-Mesh charge read-out. Top: $100 \% \mathrm{CF}_{4}$; Bottom: $12.5 \% \mathrm{CF}_{4}+$ Helium.

The total ionization left by the track is measured in three ways: (1) integrating the light in the CCD track, (2) measuring the pulse peak height of the central anode signal and (3) integrating the current signal from the mesh channel. At the drift voltage used in these data, the $\alpha$ tracks saturate the digitizer of the ground mesh and possibly the anode preamp. Using an 8-bit ADC, it is not possible to have both a fine energy granularity and a large range. The gain is optimized to have good energy resolution for low-momentum recoils created in the neutron scattering rather than the $\alpha$ studies. For this reason only methods 

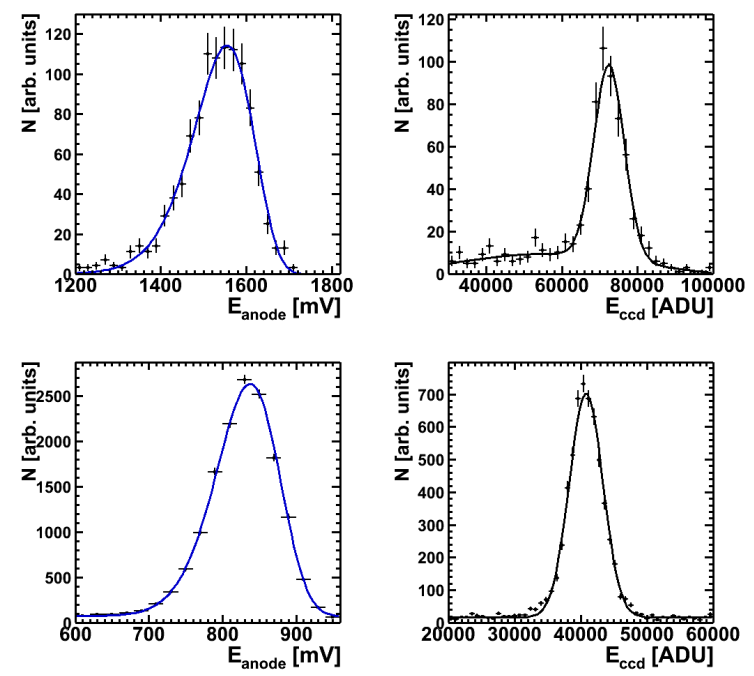

Figure 4: Energy gain calibration using the charge read-out (left) and CCD (right). for 100\% (top) and $12.5 \%$, (bottom) $\mathrm{CF}_{4}$ mixtures.

(1) and (2) are used for determining track energy. Electron attachment was measured to be negligible at our drift fields [16].

The energy distributions from the anode and CCD are shown for two gas mixtures in Fig. 4. We have fit the peak position for the energy calibration. The results of the calibration are given in Table 1. The energy is quoted in units of alpha equivalent energy to denote that the detector response is calibrated with an alpha source. We do not apply corrections due to ionization quenching at lower recoil momenta due to lack of experimental data.

The gas gain shown in Table 1 is calculated from the charge energy calibration, the preamplifier gain of $1.5 \mathrm{mV} / \mathrm{pC}$, and the gas work function. The work function of $\mathrm{CF}_{4}$ gas was measured to be $33.8 \mathrm{eV}$ [17]. This is used for calculating the gain of all mixtures.

\section{The Neutron Source Run}

The response of the detector to neutrons was characterized using a $1.24 \mathrm{mCi}$ ${ }^{252} \mathrm{Cf}$ source. ${ }^{252} \mathrm{Cf}$ decays via spontaneous fission $3 \%$ of the time emitting multiple neutrons with a mean energy of $2.35 \mathrm{MeV}$ [18. Given the activity of this source, we expect $5.7 \times 10^{6}$ neutrons per second in all directions. The source is placed in a collimator made of borated plastic, $2.1 \mathrm{~m}$ from the detector. The high flux of both neutrons and x-rays causes an increase in the rate of sparks in the TPC from $\sim 0.01 \mathrm{~Hz}$ to $0.1 \mathrm{~Hz}$. A spark is identified as an image with a mean number of ADU $1 \%$ greater than the previous image. Events within 6 

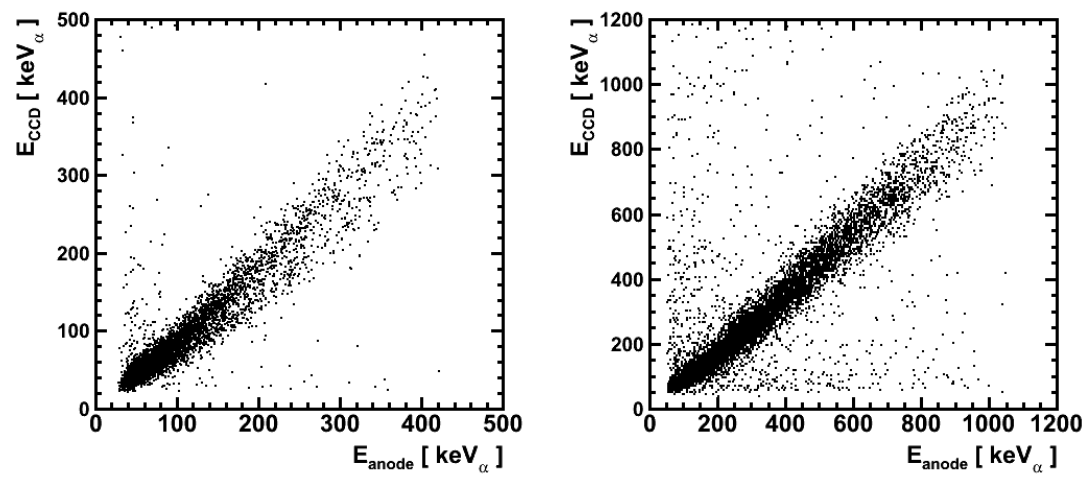

Figure 5: To validate the $\alpha$ source calibration, we use CCD light versus charge read-out signal for neutron scatters from a ${ }^{252} \mathrm{Cf}$ source. Left: $100 \% \mathrm{CF}_{4}$. Right: $12.5 \% \mathrm{CF}_{4}+\mathrm{He}$.

\begin{tabular}{l|c}
\hline \hline \% of $\mathrm{CF}_{4}$ & Requirement \\
\hline All cases & Two adjacent pixels with counts $>3.7 \sigma$ above mean [2]; \\
& Tracks are contained within $24 \leq x, y \leq 1000$ pixels; \\
& Maximum pixel $<250$ ADU and $<25 \%$ of total signal; \\
& Only one track in the image; \\
& No two tracks within 12 pixels in 1 run \\
\hline $100 \%$ & Tracks shorter than 80 pixels $(1.3 \mathrm{~cm})$ \\
\hline \hline
\end{tabular}

Table 2: Cuts applied to CCD images for the study.

exposures of a spark are excluded from analysis to allow sufficient time for the high voltage to recover.

\subsection{CCD, Charge and Matching Cuts}

The cuts to isolate good events are applied to the CCD image, the chargereadout signal and the charge-light matching. For each run condition described in Table 1, we identified a set of optimized cuts. Table2 describes the cuts placed on CCD images. Only cuts on track-length are gas-specific. The other cuts are designed to restrict tracks to the fiducial volume and remove backgrounds from ionization within the CCD chip, noise artifacts, and hot pixels.

The charge-readout cuts are more gas dependent than the CCD cuts. They can be divided into rise time cuts and pulse height cuts, summarized in Tables 3 and 4 respectively. For the veto we define two variables: the time is takes the pulse to rise from $10 \%$ to $90 \%$ of the peak pulse height, $t_{v e t o}$, and the ratio of the mesh peak pulse height to the veto peak pulse height, $R_{\text {veto }}$. For the mesh and anode, we define pulse rise times, $t_{m e s h}$ and $t_{\text {anode }}$. The pulse height range over which $t_{\text {mesh }}$ and $t_{\text {anode }}$ are defined is tuned for each gas mixture. 


\begin{tabular}{l|c|c|c}
\hline \hline \% of $\mathrm{CF}_{4}$ & $t_{\text {veto }}$ & $t_{\text {anode }}$ and $t_{\text {mesh }}$ & Pulseheight Range \\
\hline $100 \%$ & $<800 \mathrm{~ns}$ & $t_{\text {mesh }}<30 \mathrm{~ns}$ for pulseheight $<100 \mathrm{mV}$ & $25 \%$ to $75 \%$ \\
& & $t_{\text {mesh }}<25 \mathrm{~ns}$ for pulseheight $>100 \mathrm{mV}$ & $25 \%$ to $75 \%$ \\
\hline $12.5 \%$ & $<800 \mathrm{~ns}$ & $t_{\text {anode }}<1250 \mathrm{~ns}$ & $10 \%$ to $90 \%$ \\
\hline \hline
\end{tabular}

Table 3: Rise time requirements. Variables are defined in the text. Events are accepted if they pass the requirements listed. The pulseheight range over which $t_{\text {veto }}$ is measured is always $10 \%$ to $90 \%$. The pulseheight range for the definition of $t_{\text {mesh }}$ and $t_{\text {anode }}$ vary as listed in column 4 .

\begin{tabular}{l|c|c|c|c}
\hline \hline$\%$ of $\mathrm{CF}_{4}$ & $R_{\text {veto }}$ & $R_{\text {elec }}$ & $R_{\text {ion }}$ & $R_{\text {mesh }}$ \\
\hline $100 \%$ & $>4$ & $1.7<R_{\text {elec }}<2.7$ & $1.9<R_{\text {ion }}<3.5$ & $\mathrm{~N} / \mathrm{A}$ \\
$12.5 \%$ & $>3$ & N/A & N/A & $1.3<R_{\text {mesh }}<3$ \\
\hline \hline
\end{tabular}

Table 4: Pulse height ratio requirements. The ratios are defined in the text. Events are accepted if they pass the requirements listed in this table. N/A indicates that this variable is not used.

For the mesh we also define two ratios for the pulse height: $R_{\text {elec }}$ the ratio of the electron peak mesh pulse height to the height of the anode pulse and $R_{i o n}$ the ratio of the ion peak mesh pulse height to the anode pulse height. $R_{m e s h}$ is a simple ratio of the anode pulse height to the mesh pulse height.

The CCD and charge readout run autonomously. Matching the signals proceeds by comparing the CCD track energy to the charge energy, where the best energy match is identified as the true signal from the track. The requirements for matching are shown in Table 5 . Optical effects are estimated by measuring the mean ratio of the energies of the light and charge signals in neutron calibration running as a function of the distance from the image center. This is used to correct the energy of the light signal to obtain a linear relationship between the two energy measurements. The energy from the anode channel is more accurate than the CCD energy, so it is used as the final track energy. Neutron source data shown in Fig. 5 confirms a linear relationship between the summed anode energy and the CCD energy, demonstrating a good match between a CCD track and an anode waveform can be found for most of the events.

The efficiency of the cuts, including charge-light matching for neutron events

\begin{tabular}{l|c}
\hline \hline \% of $\mathrm{CF}_{4}$ & Requirement \\
\hline $100 \%$ & $|\Delta E|<40 \mathrm{keV}_{\alpha}$ if $E_{\text {anode }}<150 \mathrm{keV}_{\alpha} ;$ \\
& $|\Delta E|<75 \mathrm{keV}_{\alpha}$ if $E_{\text {anode }}>150 \mathrm{keV}_{\alpha} ;$ \\
\hline $12.5 \%$ & $|\Delta E|<75 \mathrm{keV}_{\alpha}$ for $E_{\text {anode }}<250 \mathrm{keV}_{\alpha} ;$ \\
& $|\Delta E|<125 \mathrm{keV}_{\alpha}$ for $250<E_{\text {anode }}<500 \mathrm{keV}_{\alpha} ;$ \\
& $|\Delta E|<150 \mathrm{keV}_{\alpha}$ for $E_{\text {anode }}>500 \mathrm{keV}_{\alpha}$ \\
\hline \hline
\end{tabular}

Table 5: Requirements for CCD and charge event matching in this study, where $|\Delta E|=$ $\left|E_{C C D}-E_{\text {anode }}\right|$. 

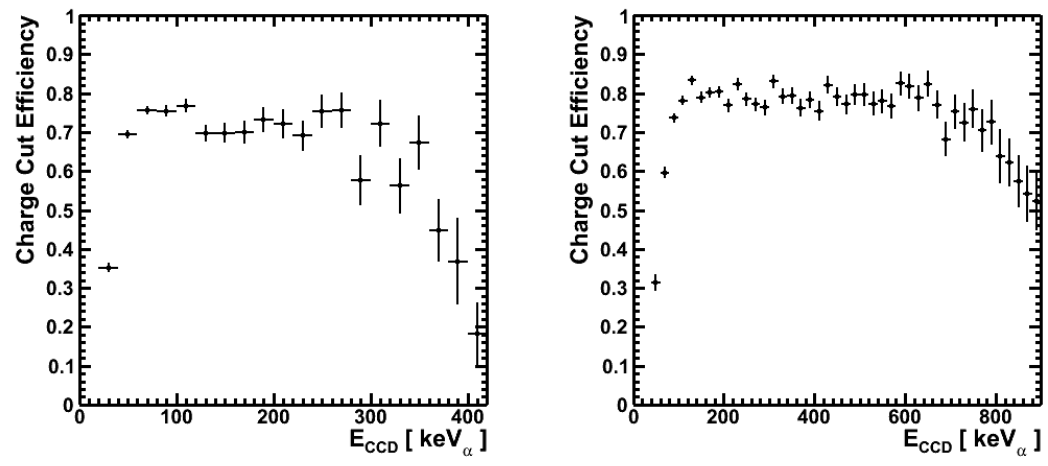

Figure 6: Efficiency of charge analysis in accepting tracks identified by the CCD analysis. The efficiency plateaus at $70-80 \%$ are due to exposure difference between the charge and light readout and depend on the event readout time. The decrease in efficiency at higher energies is from the mesh pulse reaching the maximum value read out by the digitizer. Left: $100 \%$ $\mathrm{CF}_{4}$ gas. Right: $12.5 \% \mathrm{CF}_{4}+\mathrm{He}$ mixture. The errors on the data points reflect the statistics of the calibration run.

is given in Fig. 6. This data is dominated by nuclear recoils rather than CCD artifacts, so is ideal for the efficiency determination. The efficiency is defined as the ratio of the number of tracks passing all CCD cuts, charge cuts and chargelight matching compared to the number of CCD tracks found without using the charge signals.

\subsection{Discussion of Neutron Source Run}

The neutron source generates a large number of nuclear recoils, with $\mathcal{O}(1)$ neutron-induced nuclear recoils detected per $1 \mathrm{~s}$ exposure. CCD images and charge waveforms of neutron-induced recoils in two events with different gas mixtures are shown in Fig. 7. A large number of electronic recoils from the high $\gamma$ flux are observed in the charge signals. However the CCD remains blind to these events due to their low primary ionization density. The source creates a much higher number of CCD-induced artifacts, likely from electronic recoils inside the active volume of the CCD chip. Even with these large source activity issues, the cuts are sufficient for this analysis.

After applying all cuts, we use the measured track angles and ranges, Fig. 8 and Fig. 9, to evaluate the performance of the detector and the analysis. The measured two-dimensional track range generally falls just below the expected mean three-dimensional range calculated with the publicly available program SRIM [19]. For the $12.5 \% \mathrm{CF}_{4}$ mixture, the measured two-dimensional track range falls close to the expected range for helium tracks. This follows from the fact that helium recoils are kinematically favored across most of the energy range measured here. At lower energies, where both helium and fluorine recoils are expected, the track ranges appear to be consistent with either nucleus. 

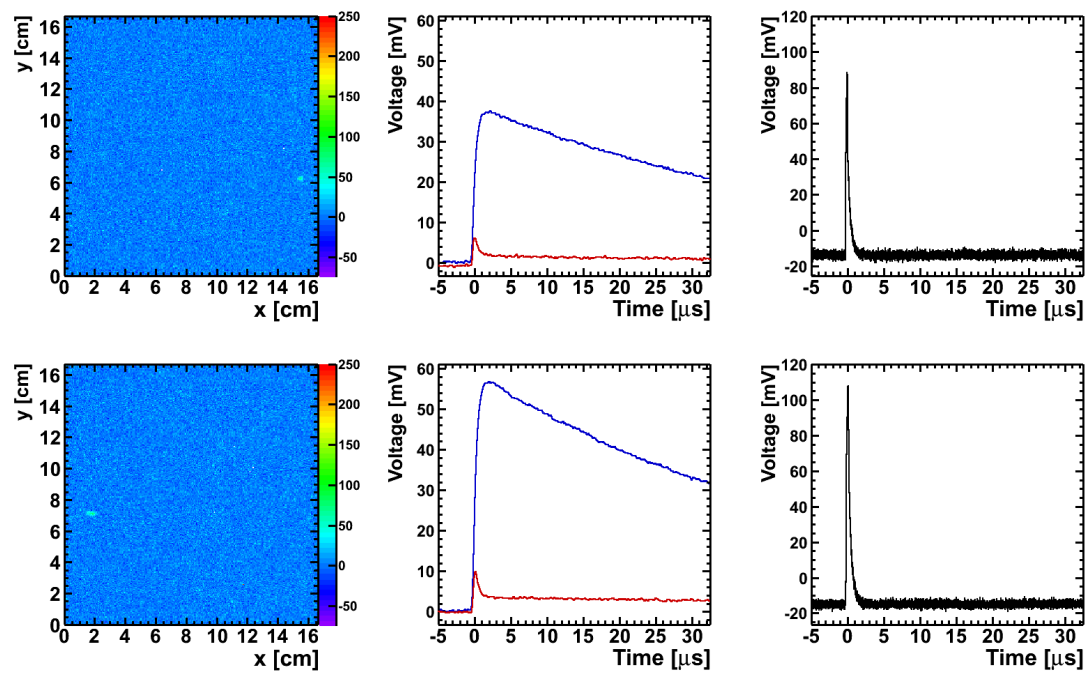

Figure 7: Example figures of neutron recoils from ${ }^{252} \mathrm{Cf}$ source showing graphical images of CCD and charge read-out system. Left: CCD image of ${ }^{4} \mathrm{He}$ recoil. Middle: Waveform from the Veto and Anode charge read-out. Right: Waveform from the Ground-Mesh charge read-out. Top row is $100 \% \mathrm{CF}_{4}$ and bottom is $12.5 \% \mathrm{CF}_{4}$.

We use the track angles to reconstruct the location of the neutron source. Tracks along the direction of the incoming neutrons should have a decreasing light profile, and this "head-tail effect" can be used to reconstruct the sense of track direction [20]. Two peaks are seen in the angular distribution of Fig. 8 The larger peak at -90 degrees is along the true mean direction of the nuclear recoils. The second peak at +90 degrees is primarily from events where the track direction was mis-reconstructed. The large width of the distribution is expected from elastic scattering kinematics. Additionally, some nuclear recoils are from neutrons that have scattered several times within the laboratory, and no longer appear to originate at the source position.

A model of helium and fluorine recoils is fitted to the measured recoil spectrum in Fig. 10. The fit uses the likelihood approach with the probability density functions for helium and fluorine based on simulation, and a ${ }^{252} \mathrm{Cf}$ spectrum based on ENDF tables [13. We find the fraction of helium recoil events to be $61 \pm 1 \%$. Another way to present this result is in terms of the unfolded neutron spectrum. We use the recoil data and the unfolding matrix, based on the simulation, to obtain the neutron spectrum as shown in Fig. 10. There is a reasonable agreement with the neutron spectrum from a ${ }^{252} \mathrm{Cf}$ source that is based on ENDF tables 13. In order to improve the agreement between the data and the simulation of the recoil and neutron spectra, we need to improve the simulation to include the efficiency loss due to sparks. This requires additional measurements, and a full simulation of the detector material which modifies the 

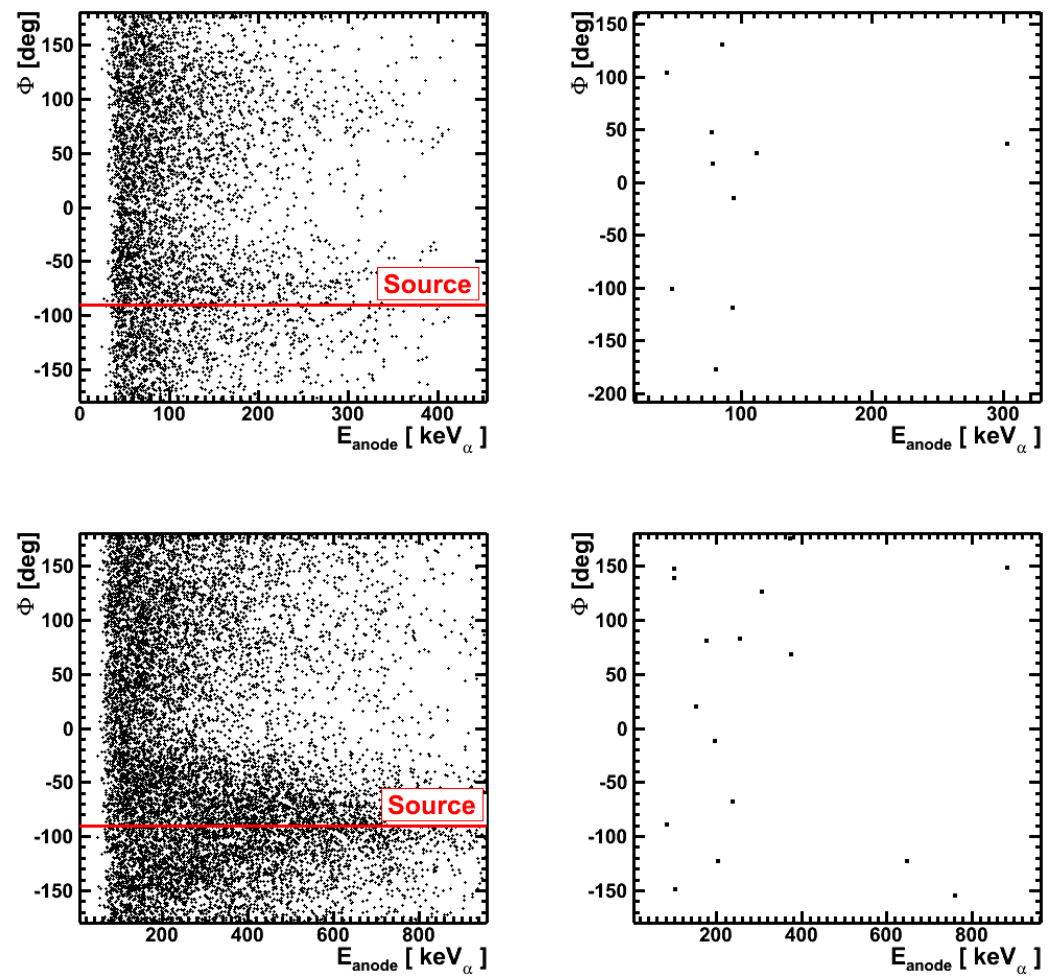

Figure 8: Energy-Angular distribution for calibration and background runs for two gas mixtures. Left: Calibration run with ${ }^{252} \mathrm{Cf}$ source. Right: Background run. Top: $100 \% \mathrm{CF}_{4}$ gas. Bottom: $12.5 \% \mathrm{CF}_{4}$. The direction of the neutrons from the source is indicated by a red solid line at -90 degrees. The population at +90 degrees is due to incorrect reconstruction of the track direction.

energy spectrum of neutrons entering the sensitive region.

\section{Surface Run}

With the DCTPC prototype, we have also taken data with no sources to measure cosmogenic, environmental and detector backgrounds in a surface lab on the MIT campus. The lab is on the ground floor of a two-story building. The lab has approximately $1 \mathrm{~m}$ concrete walls on all sides. After removing spark events, we obtain 112713 exposures in pure $\mathrm{CF}_{4}$ and 103822 exposures in the $12.5 \% \mathrm{CF}_{4}$ mixture. As in the other runs, each exposure is $1 \mathrm{~s}$, so this corresponds to 1.3 and 1.2 days of exposure, respectively.

In the pure $\mathrm{CF}_{4}$ run, we identified 128 possible tracks in the $\mathrm{CCD}$ analysis. Most of these tracks appear to be from CCD backgrounds such as noise artifacts 

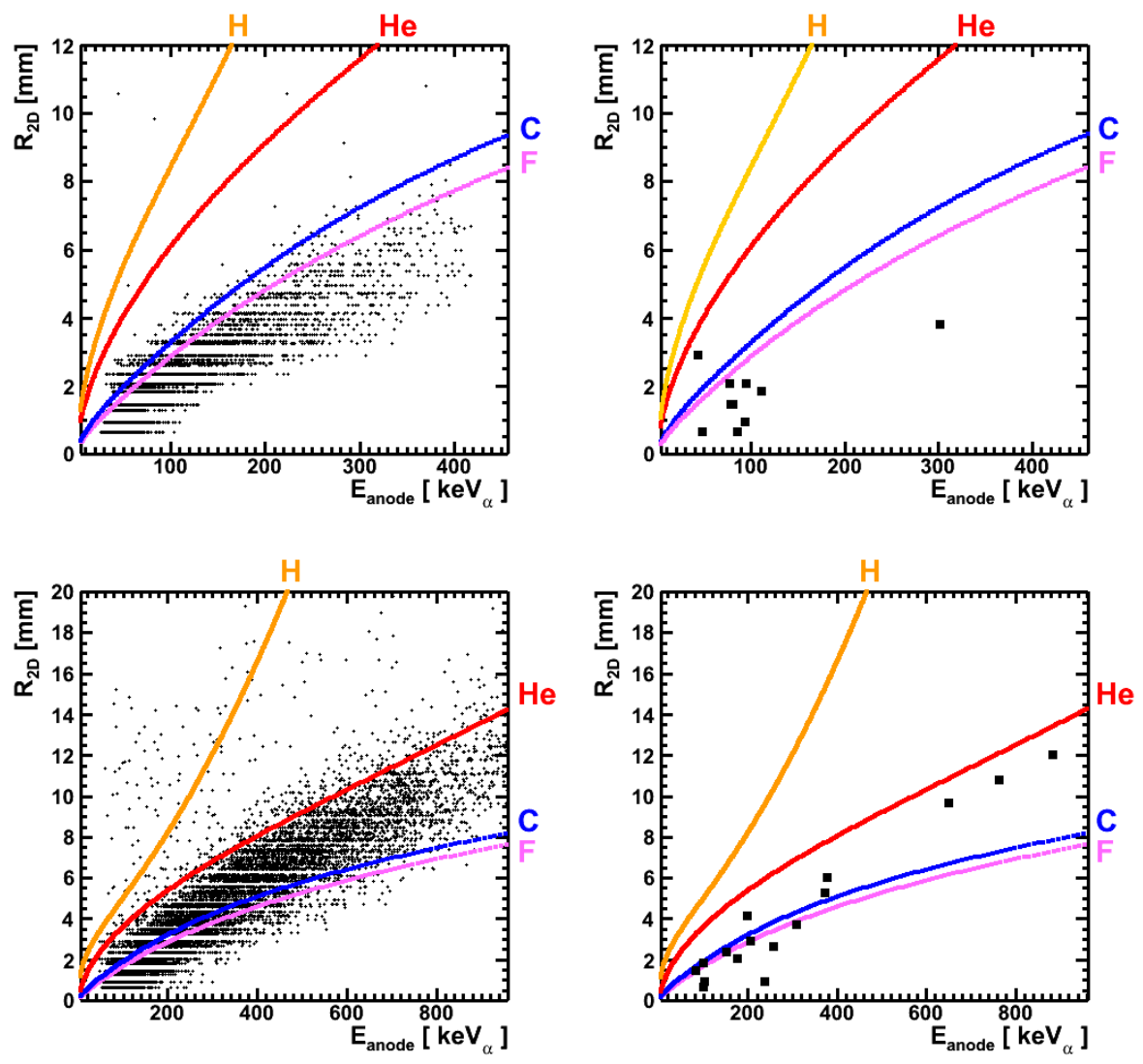

Figure 9: Energy-Range distribution, calculated in two dimensions, for calibration and background runs with two different gas mixtures. Left: Calibration run with ${ }^{252} \mathrm{Cf}$ source. Right: Background run. Top: $100 \% \mathrm{CF}_{4}$ gas. Bottom: $12.5 \% \mathrm{CF}_{4}$ mixture. Three-dimensional analytical prediction of the $\mathrm{dE} / \mathrm{dx}$ curve for different nuclear elements of the gas mixture is shown in colored lines. 

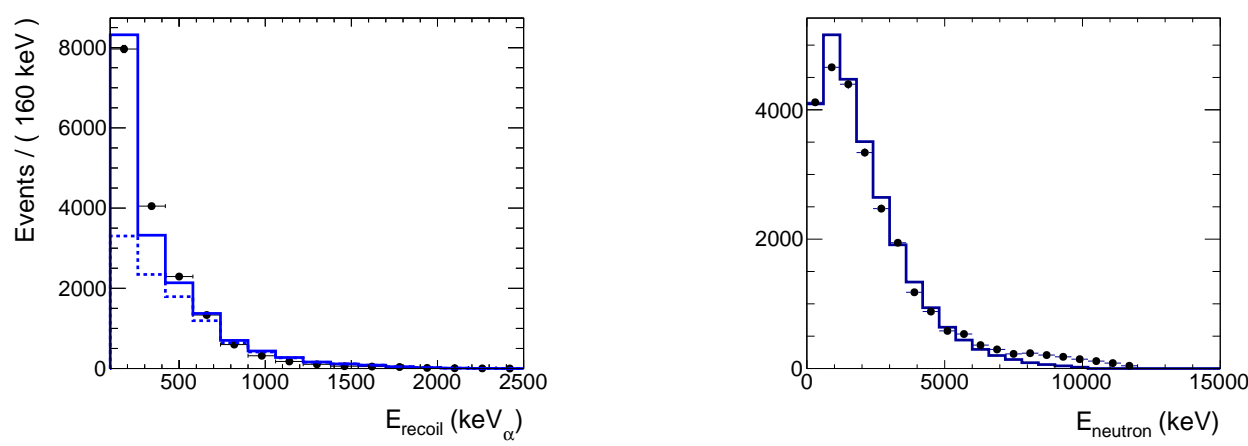

Figure 10: Left: A fit to a recoil spectrum (markers) using a likelihood model based on helium and fluorine recoils. The helium contribution (dashed) and total recoil distribution (full line) are shown as histograms. Right: Unfolded neutron spectrum from a calibration run with ${ }^{252} \mathrm{Cf}$ source (markers), and a spectrum based on the ${ }^{252} \mathrm{Cf}$ table [13] (histogram).

or ionization in the CCD. Of these 128 tracks, only 10 were matched to a charge trigger. Several additional tracks had too much energy to be accurately measured by the charge channels, although the pulses were collected. These tracks are mostly low energy $\left(E<100 \mathrm{keV}_{\alpha}\right)$ and the ranges generally appear to be consistent with the expected values from SRIM for fluorine recoils. A single event has a range more consistent with helium or hydrogen tracks.

In the $12.5 \%$ mixture run, we identified 71 potential tracks in the CCD analysis, with 16 having a matching charge signal. As with the pure $\mathrm{CF}_{4}$ run, most of the potential tracks seen in the CCD are likely noise artifacts or tracks left directly in the silicon of the CCD chip. As expected, we see more tracks in this run due to the presence of helium. The tracks seen here are also generally at higher energies than were seen in pure $\mathrm{CF}_{4}$. This is to be expected if most of the tracks come from elastic scattering from neutrons due to the favorable kinematics of neutron-helium scattering compared to neutron-fluorine scattering. Fewer events at lower energies are seen due to the differing energy thresholds of the two gas mixtures. There does not appear to be a favored direction of these tracks, although much higher statistics would be necessary to confirm this. The events with $E>300 \mathrm{keV}_{\alpha}$ here have ranges consistent with helium recoils but not carbon or fluorine, while most of the other tracks have ranges consistent with either carbon or fluorine nuclei or near vertical helium nuclei.

\section{Future Underground Measurement}

The first generation detector is being installed in the far-hall of Double Chooz at 300 m.w.e. We can use the 13 neutrons per day measured in the surface run to make an estimate for our event rate in the far-hall. For the surface run, we measure a muon rate of 100 muons per $\mathrm{m}^{2} \mathrm{~s}^{1}$. We assume the mean muon energy is $\sim 3 \mathrm{GeV}$. The muon rate in the far-hall is 0.4 muons per $\mathrm{m}^{2} \mathrm{~s}^{1}$ with 


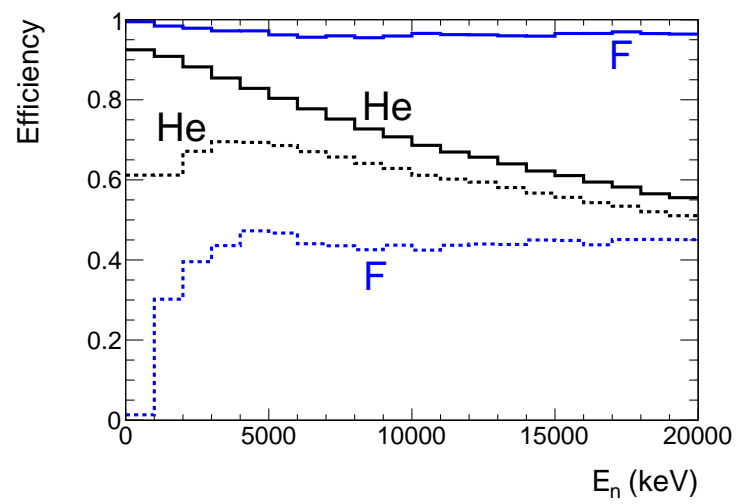

Figure 11: The efficiency for reconstructing helium and fluorine recoils as a function of neutron energy. The geometric efficiency (full line) requires full track containment in the CCD view-field. The full efficiency (dashed line) assumes a step-function for the recoil reconstruction efficiency with thresholds given in the text.

a mean muon energy of $60 \mathrm{GeV}$ [4. Using the power law scaling from Ref. [11, we estimate $\sim 0.5$ events per day.

A study of the detector efficiency, shown in Fig. 11, indicates that events collected in this run is expected to sensitive to to neutrons from $(\alpha, \mathrm{n})$ and muon interactions. Fluorine and helium recoils are efficiently reconstructed above 150 and $100 \mathrm{keV}$ of recoil energy, respectively. Since fluorine recoils are shorter, they have a high probability to fit within CCD view-field. Geometric acceptance efficiencies are shown as full lines in Fig. 11. Helium recoils receive more energy from a collision with neutrons and leave longer tracks - a combination that is favored by reconstruction algorithms. Helium recoils are more efficient at energies $\lesssim 20 \mathrm{MeV}$. Fluorine appears more efficient if the detector is being used for neutrons with the energy of tens of MeV's due to more compact tracks.

\subsection{Conclusion}

We have modified the DMTPC dark matter detector design to produce a 2.8 liter, first-generation directional neutron detector. The primary change to the DMTPC design was to add helium as a target for the neutrons. We have demonstrated that a gas mixture of 75 Torr of $\mathrm{CF}_{4}$ and 525 Torr of helium is capable of extracting the energy and angular distributions of the neutrons from a ${ }^{252} \mathrm{Cf}$ source.

The sensitivity to higher energy neutrons and an estimate of 0.5 events per day at the Double Chooz far site indicates that the first generation detector could make an interesting measurement at the far site on its own. The success of this detector motivates the construction of a full-sized DCTPC system. This will consist of two $60 \mathrm{l}$ detectors, located at the near and far halls of Double Chooz, which can be interchanged for comparison of systematics. The goal is 
to measure the neutron flux, with information on both energy and direction, as a function of time, in order to tune the fast neutron simulation.

\section{Acknowledgments}

The authors thank Prof. Peter Fisher, of MIT, and the Double Chooz collaboration for valuable input. JMC, KT and LW are supported by the National Science Foundation. DD is supported by the Department of Energy. JL is supported by a Massachusetts Institute of Technology Lyons Fellowship and the Institute for Soldier Nanotechnology.

\section{References}

[1] J. A. Formaggio and C. J. Martoff. Backgrounds to sensitive experiments underground. Ann. Rev. Nucl. Part. Sci., 54:361-412, 2004.

[2] S. Ahlen et al. First Dark Matter Search Results from a Surface Run of the 10-L DMTPC Directional Dark Matter Detector. Phys. Lett., B695:124$129,2011$.

[3] Alvaro Roccaro et al. A Background-Free Direction-Sensitive Neutron Detector. Nucl. Instrum. Meth., A608:305-309, 2009.

[4] F. Ardellier et al. Double Chooz: A search for the neutrino mixing angle $\theta_{13} .2006$.

[5] Dongming Mei and A. Hime. Muon-Induced Background Study for Underground Laboratories. Phys. Rev., D73:053004, 2006.

[6] A. Ferrari, P. R. Sala, A. Fasso, and J. Ranft. FLUKA: A multi-particle transport code (Program version 2005). CERN-2005-010.

[7] A. Fasso et al. The Physics Models of FLUKA: Status and Recent Development. 2003.

[8] John Allison et al. Geant4 developments and applications. IEEE Trans. Nucl. Sci., 53:270, 2006.

[9] S. Agostinelli et al. GEANT4: A simulation toolkit. Nucl. Instrum. Meth., A506:250-303, 2003.

[10] B. Armbruster et al. Upper limits for neutrino oscillations $\bar{\nu}_{\mu}$ to $\bar{\nu}_{e}$ from muon decay at rest. Phys. Rev., D65:112001, 2002.

[11] S. Abe et al. Production of Radioactive Isotopes through Cosmic Muon Spallation in KamLAND. Phys. Rev., C81:025807, 2010.

[12] M. Aglietta et al. Measurement of the neutron flux produced by cosmic-ray muons with LVD at Gran Sasso. 1999. 
[13] M.B. Chadwick et al. ENDF/B-VII.0: Next Generation Evaluated Nuclear Data Library for Nuclear Science and Technology. Nuclear Data Sheets, 107:2931-3060, 2006.

[14] D. Dujmic et al. Charge Amplification Concepts for Direction-Sensitive Dark Matter Detectors. Astropart. Phys., 30:58-64, 2008.

[15] H. Yegoryan. Study of Alpha Background in a Dark Matter Detector. Senior Thesis, Massachusetts Institute of Technology, 2010.

[16] T. Caldwell et al. Transport properties of electrons in CF(4). 2009.

[17] I.C. Wolfe. Measurement of Work Function in $\mathrm{CF}_{4}$ Gas. Senior Thesis, Massachusetts Institute of Technology, 2010.

[18] K. Nakamura et al. The Rev. of Part. Physics. J. Phys., G37:075021, 2010.

[19] J.F. Ziegler, J.P. Biersack, and M.D. Ziegler. SRIM: The Stopping and Range of Ions in Matter. http://www.srim.org/, 2011.

[20] D. Dujmic et al. Observation of the 'head-tail' effect in nuclear recoils of low-energy neutrons. Nucl.Instrum.Meth., A584:327-333, 2008. 